\title{
Estimation of general parameter in adaptive cluster sampling using two auxiliary variables
}

\author{
Faryal Younis* and Javid Shabbir \\ Department of Statistics, Quaid-i-Azam University, Islamabad, Pakistan.
}

\begin{abstract}
In this article, exponential ratio type estimators are proposed for general parameter in adaptive cluster sampling. The estimators utilise information on two auxiliary variables in three different situations, i.e. partial, no and full information about population parameters of auxiliary variables. The proposed estimators for general parameter can be used to estimate population mean, coefficient of variation, standard deviation and variance of the variable of interest. The bias and mean square error equations for the proposed estimators are derived using first order approximation. The proposed estimators are more efficient than usual sample estimators and ratio estimators in all three situations under adaptive cluster sampling. Two different populations are used for numerical illustration.
\end{abstract}

Keywords: Adaptive cluster sampling, auxiliary variable, coefficient of variation.

\section{INTRODUCTION}

In the theory of survey sampling, estimation of different population parameters is widely considered using several sampling designs. Conventional sampling designs may not be helpful when the population under consideration is rare and clustered. Thompson (1990) introduced adaptive cluster sampling for such situations as an alternative. The scheme provides higher sampling yields for highly clumped, sporadic and hidden populations.

Adaptive cluster sampling is valuable for studies on drug addicts, endangered species of animals, contagious diseases, rare and precious plants, minerals and natural resources. In adaptive cluster sampling, an initial sample is selected by some conventional sampling design. All the selected units are examined for a predefined condition $C$. If any of these units satisfy the condition, its neighbouring units are added to the sample. Further, if any of these newly added units satisfy the condition, its neighbouring units are also included in the sample and the process continues until no more units satisfy the condition. Estimators for general parameter under three different situations are proposed.

1. When population mean and variance are known for one auxiliary variable (say $z$ ) and unknown for the other auxiliary variable (say $x$ ).

2. When population mean and variance are unknown for both auxiliary variables.

3. When population mean and variance are known for both auxiliary variables.

When population parameters for at least one of the auxiliary variable are unknown, we adopt the two phase sampling scheme using adaptive cluster sampling at each phase as follows:

1. In phase one, a large sample of size $n^{\prime}$ is drawn using adaptive cluster sampling based on auxiliary variable and unknown parameters of auxiliary variables $(x$ and $z$ ) are estimated.

2. In phase two, network structure of the phase one sample is used to select a sample of size $n\left(n<n^{\prime}\right)$ using adaptive cluster sampling based on the study

*Corresponding author (faryalyounis@gmail.com; (D https://orcid.org/0000-0002-7314-3227)

This article is published under the Creative Commons CC-BY-ND License (http://creativecommons.org/licenses/by-nd/4.0/). This license permits use, distribution and reproduction, commercial and non-commercial, provided that the original work is properly cited and is not changed in anyway. 
variable. Thus information on the study variable $(y)$ and auxiliary variables $(x$ and $z)$ is observed.

Consider a finite population of $N$ units with labels $1,2, \ldots, N$. Let $y_{i}$ and $\left(x_{i}, z_{i}\right)$ be the observed values of the study and the auxiliary variables and $w_{y_{i}}$ and $\left(w_{x_{i}}, w_{z_{i}}\right)$ be the network level means. Also let $\bar{w}_{y}$ and $\left(\bar{w}_{x}, \bar{w}_{z}\right)$ be the sample means and $s_{w_{y}}^{2}$ and $\left(s_{w_{x}}^{2}, s_{w_{z}}^{2}\right)$ be the sample variances of study and auxiliary variables. Let $\bar{Y}_{w}$ and $\left(\bar{X}_{w}, \bar{Z}_{w}\right)$ and $S_{w_{y}}^{2}$ and $\left(S_{w_{x}}^{2}, S_{w_{z}}^{2}\right)$ be the corresponding population means and variances respectively, in transformed population.

The following notations are used:

$$
\begin{aligned}
& \bar{w}_{y}=\frac{1}{n} \sum_{i=1}^{n} w_{y_{i}}, \bar{w}_{x}=\frac{1}{n} \sum_{i=1}^{n} w_{x_{i}}, \bar{w}_{z}=\frac{1}{n} \sum_{i=1}^{n} w_{z_{i}}, \\
& w_{x_{i}}=\frac{1}{m_{i}} \sum_{j \in \Psi_{i}} x_{j}, w_{z_{i}}=\frac{1}{m_{i}} \sum_{j \in \Psi_{i}} z_{j}, \\
& \bar{Y}_{w}=\frac{1}{N} \sum_{i=1}^{N} w_{y_{i}}, \bar{X}_{w}=\frac{1}{N} \sum_{i=1}^{N} w_{x_{i}}, \bar{Z}_{w}=\frac{1}{N} \sum_{i=1}^{N} w_{z_{i}}, \\
& \bar{w}_{x}^{\prime}=\frac{1}{n^{\prime}} \sum_{i=1}^{n^{\prime}} w_{x_{i}}, \bar{w}_{z}^{\prime}=\frac{1}{n^{\prime}} \sum_{i=1}^{n^{\prime}} w_{z_{i}},
\end{aligned}
$$$$
s_{w_{y}}^{2}=\frac{1}{n-1} \sum_{i=1}^{n}\left(w_{y_{i}}-\bar{w}_{y}\right)^{2}, s_{w_{x}}^{2}=\frac{1}{n-1} \sum_{i=1}^{n}\left(w_{x_{i}}-\bar{w}_{x}\right)^{2},
$$$$
s_{w_{z}}^{2}=\frac{1}{n-1} \sum_{i=1}^{n}\left(w_{z_{i}}-\bar{w}_{z}\right)^{2},
$$$$
s_{w_{x}}^{2^{\prime}}=\frac{1}{n^{\prime}-1} \sum_{i=1}^{n^{\prime}}\left(w_{x_{i}}-\bar{w}_{x}^{\prime}\right)^{2}, s_{w_{z}}^{2^{\prime}}=\frac{1}{n^{\prime}-1} \sum_{i=1}^{n^{\prime}}\left(w_{z_{i}}-\bar{w}_{z}^{\prime}\right)^{2},
$$$$
S_{w_{y}}^{2}=\frac{1}{N-1} \sum_{i=1}^{N}\left(w_{y_{i}}-\bar{Y}_{w}\right)^{2} \text {, }
$$$$
S_{w_{x}}^{2}=\frac{1}{N-1} \sum_{i=1}^{N}\left(w_{x_{i}}-\bar{X}_{w}\right)^{2}, S_{w_{z}}^{2}=\frac{1}{N-1} \sum_{i=1}^{N}\left(w_{z_{i}}-\bar{Z}_{w}\right)^{2},
$$$$
C_{w_{y}}=\frac{S_{w_{y}}}{\bar{Y}_{w}}, C_{w_{x}}=\frac{S_{w_{x}}}{\bar{X}_{w}},
$$

where,
$C_{w_{z}}=\frac{S_{w_{z}}}{\bar{Z}_{w}}, C_{w_{y} w_{x}}=\rho_{w_{y} w_{x}} C_{w_{y}} C_{w_{x}}, C_{w_{x} w_{z}}=$

$\rho_{w_{x} w_{z}} C_{w_{x}} C_{w_{z}}, C_{w_{y} w_{z}}=\rho_{w_{y} w_{z}} C_{w_{y}} C_{w_{z}}$.

The following error terms are used:

$e_{0}=\frac{\bar{w}_{y}-\bar{Y}_{w}}{\bar{Y}_{w}}, e_{1}=\frac{\bar{w}_{x}-\bar{X}_{w}}{\bar{X}_{w}}, e_{1}^{\prime}=\frac{\bar{w}_{x}^{\prime}-\bar{X}_{w}}{\bar{X}_{w}}$,

$e_{2}=\frac{\bar{w}_{z}-\bar{Z}_{w}}{\bar{Z}_{w}}, e_{2}^{\prime}=\frac{\bar{w}_{z}^{\prime}-\bar{Z}_{w}}{\bar{Z}_{w}}$,

$e_{3}=\frac{S_{w_{y}}^{2}-S_{w_{y}}^{2}}{S_{w_{y}}^{2}}, e_{4}=\frac{s_{w_{x}}^{2}-S_{w_{x}}^{2}}{S_{w_{x}}^{2}}, e_{4}^{\prime}=\frac{S_{w_{x}}^{2^{\prime}}-S_{w_{x}}^{2}}{S_{w_{x}}^{2}}$,

$e_{5}=\frac{S_{w_{z}}^{2}-S_{w_{z}}^{2}}{S_{w_{z}}^{2}}, e_{5}^{\prime}=\frac{S_{w_{z}}^{2^{\prime}}-S_{w_{z}}^{2}}{S_{w_{z}}^{2}}$,

such that $E\left(e_{i}\right)=0 \quad \forall i=0,1,2,3,4,5$ and $E\left(e_{i}^{\prime}\right)=$ $0 \forall i=1,2,4,5$.

Also,

$E\left(e_{0}^{2}\right)=\theta C_{w_{y}}^{2}, E\left(e_{2} e_{3}\right)=\theta \lambda_{201} C_{w_{z}}, E\left(e_{1}^{\prime} e_{2}^{\prime}\right)=E\left(e_{1} e_{2}^{\prime}\right)$

$=E\left(e_{2} e_{1}^{\prime}\right)=\theta^{\prime} C_{w_{x} w_{z}}$,

$E\left(e_{1}^{2}\right)=\theta C_{w_{x}}^{2}, E\left(e_{2} e_{4}\right)=\theta \lambda_{021} C_{w_{z}}, E\left(e_{1}^{\prime} e_{4}^{\prime}\right)=E\left(e_{1} e_{4}^{\prime}\right)$

$=E\left(e_{4} e_{1}^{\prime}\right)=\theta^{\prime} \lambda_{030} C_{w_{x}}$,

$E\left(e_{2}^{2}\right)=\theta C_{w_{z}}^{2}, E\left(e_{1} e_{4}\right)=\theta \lambda_{030} C_{w_{x}}, E\left(e_{1}^{\prime} e_{5}^{\prime}\right)=E\left(e_{1} e_{5}^{\prime}\right)$

$=E\left(e_{5} e_{1}^{\prime}\right)=\theta^{\prime} \lambda_{012} C_{w_{x}}$,

$E\left(e_{3}^{2}\right)=\theta \lambda_{400}^{*}, E\left(e_{0} e_{5}\right)=\theta \lambda_{102} C_{w_{y}}, E\left(e_{2}^{\prime} e_{4}^{\prime}\right)=E\left(e_{2} e_{4}^{\prime}\right)$

$=E\left(e_{4} e_{2}^{\prime}\right)=\theta^{\prime} \lambda_{021} C_{w_{z}}$,

$E\left(e_{4}^{2}\right)=\theta \lambda_{040}^{*}, E\left(e_{0} e_{3}\right)=\theta \lambda_{300} C_{w_{y}}, E\left(e_{2}^{\prime} e_{5}^{\prime}\right)=E\left(e_{2} e_{5}^{\prime}\right)$

$=E\left(e_{5} e_{2}^{\prime}\right)=\theta^{\prime} \lambda_{003} C_{w_{z}}$,

$E\left(e_{5}^{2}\right)=\theta \lambda_{004}^{*}, E\left(e_{3} e_{4}\right)=\theta \lambda_{220}^{*}, E\left(e_{3} e_{5}\right)=\theta \lambda_{202}^{*}$, 


$$
\begin{aligned}
& , E\left(e_{4}^{\prime} e_{5}^{\prime}\right)=E\left(e_{4} e_{5}^{\prime}\right)=E\left(e_{5} e_{4}^{\prime}\right)=\theta^{\prime} \lambda_{022}^{*}, \\
& E\left(e_{0} e_{1}\right)=\theta C_{w_{y} w_{x}}, E\left(e_{0} e_{2}\right)=\theta C_{w_{y} w_{z}}, E\left(e_{1} e_{5}\right)=\theta \lambda_{012} C_{w_{x}} \text {, } \\
& , E\left(e_{1}^{\prime 2}\right)=E\left(e_{1} e_{1}^{\prime}\right)=\theta^{\prime} C_{w_{x}}^{2}, \\
& E\left(e_{1} e_{2}\right)=\theta C_{w_{x} w_{z}}, E\left(e_{0} e_{4}\right)=\theta C_{w_{y}} \lambda_{120}, E\left(e_{3} e_{5}^{\prime}\right)=\theta^{\prime} \lambda_{202}^{*}, \\
& \text {, } E\left(e_{2}^{\prime 2}\right)=E\left(e_{2} e_{2}^{\prime}\right)=\theta^{\prime} C_{w_{z}}^{2}, \\
& E\left(e_{4} e_{5}\right)=\theta \lambda_{022}^{*}, E\left(e_{2} e_{5}\right)=\theta \lambda_{003} C_{w_{z}}, E\left(e_{3} e_{1}^{\prime}\right)=\theta^{\prime} \lambda_{210} C_{w_{x}}, \\
& , E\left(e_{4}^{\prime 2}\right)=E\left(e_{4} e_{4}^{\prime}\right)=\theta^{\prime} \lambda_{040}^{*}, \\
& E\left(e_{3} e_{2}^{\prime}\right)=\theta^{\prime} \lambda_{201} C_{w_{z}}, E\left(e_{3} e_{4}^{\prime}\right)=\theta^{\prime} \lambda_{220}^{*}, E\left(e_{1} e_{3}\right)=\theta \lambda_{210} C_{w_{x}}, \\
& , E\left(e_{5}^{\prime 2}\right)=E\left(e_{5} e_{5}^{\prime}\right)=\theta^{\prime} \lambda_{004}^{*}, \\
& \theta=\left(\frac{1}{n}-\frac{1}{N}\right), \mu_{r s t}=\frac{1}{N-1} \sum_{i=1}^{N}\left(w_{y i}-\bar{Y}_{w}\right)^{r}\left(w_{x i}-\bar{X}_{w}\right)^{s}\left(w_{z i}-\bar{Z}_{w}\right)^{t}, \\
& \theta^{\prime}=\left(\frac{1}{n^{\prime}}-\frac{1}{N}\right), \theta^{\prime \prime}=\theta-\theta^{\prime}=\left(\frac{1}{n}-\frac{1}{n^{\prime}}\right), \lambda_{r s t}^{*}=\lambda_{r s t}-1, \lambda_{r s t} \\
& =\frac{\mu_{r s t}}{\mu_{200}^{\frac{r}{2}} \mu_{020}^{\frac{s}{2}} \mu_{002}^{\frac{t}{2}}} .
\end{aligned}
$$

The general parameter of interest is defined by,

$$
\tau(\alpha, \beta)=\bar{Y}_{w}^{\alpha} S_{w_{y}}^{\beta}, \bar{Y}_{w} \neq 0,
$$

where $\alpha$ and $\beta$ are suitably chosen constants. For different values of $\alpha$ and $\beta, \tau(\alpha, \beta)$ reduces to the following population parameters:

1. $\alpha=1, \beta=0, \tau(\alpha, \beta)=\bar{Y}_{w}$ (population mean)

2. $\alpha=0, \beta=2, \tau(\alpha, \beta)=S_{w_{y}}^{2}$ (population variance)

3. $\alpha=-1, \beta=1, \tau(\alpha, \beta)=C_{w_{y}}$ (population coefficient of variation)

Thompson (1990) proposed an unbiased estimator for population mean based on Hansen-Hurwitz estimator as,

$$
\begin{aligned}
t_{\text {om }} & =\bar{w}_{y} \\
& =\frac{1}{n} \sum_{i=1}^{n}\left(w_{y}\right)_{i},
\end{aligned}
$$

where,

$$
\left(w_{y}\right)_{i}=\frac{1}{m_{i}} \sum_{j \in \Psi_{i}} y_{j}
$$

$\Psi_{i}$ is the network that includes unit $i,\left(w_{y}\right)_{i}$ is the average of $y$ values and $m_{i}$ is the size of that network. The variance of $t_{\text {om }}$ is,

$$
V\left(t_{o m}\right)=\theta \bar{Y}_{w}^{2} C_{w y}^{2}
$$

Thompson (1992) suggested an unbiased estimator for population variance as,

$$
\begin{aligned}
t_{o v} & =s_{w_{y}}^{2} \\
& =\frac{1}{n-1} \sum_{i=1}^{n}\left(w_{y_{i}}-\bar{w}_{y}\right)^{2} .
\end{aligned}
$$

The variance of $t_{o v}$ is,

$$
V\left(t_{o v}\right)=\theta S_{w_{y}}^{4} \lambda_{400}^{*}
$$

Similarly, an estimator for coefficient of variation can be defined as,

$$
\begin{aligned}
t_{o c} & =\hat{C}_{w_{y}} \\
& =\frac{s_{w_{y}}}{\bar{w}_{y}}
\end{aligned}
$$

The bias and mean square error (MSE) of $t_{o c}$ to first order of approximation are,

$$
\operatorname{Bias}\left(t_{o c}\right)=\theta C_{w_{y}}\left(C_{w_{y}}^{2}-\frac{1}{2} C_{w_{y}} \lambda_{300}-\frac{1}{8} \lambda_{400}^{*}\right)
$$

and

$\operatorname{MSE}\left(t_{o c}\right)=C_{w_{y}}^{2} \theta\left(C_{w_{y}}^{2}+\frac{1}{4} \lambda_{400}^{*}-C_{w_{y}} \lambda_{300}\right)$

Thus, the usual estimator for the general parameter $\tau(\alpha, \beta)$ is,

$$
\begin{aligned}
t_{o} & =\hat{\tau}_{(\alpha, \beta)} \\
& =w_{y}^{\alpha} s_{w_{y}}^{\beta}
\end{aligned}
$$

The bias and MSE of $t_{o}$, to first order of approximation are,

$$
\operatorname{Bias}\left(t_{o}\right)=\tau(\alpha, \beta) \theta\left[\frac{\alpha(\alpha-1)}{2} C_{w_{y}}^{2}+\frac{\alpha \beta}{2} C_{w_{y}} \lambda_{300}+\frac{\beta(\beta-2)}{8} \lambda_{400}^{*}\right]
$$


and

$\operatorname{MSE}\left(t_{o}\right)=\tau(\alpha, \beta)^{2} \theta\left[\alpha^{2} C_{w_{y}}^{2}+\frac{\beta^{2}}{4} \lambda_{400}^{*}+\alpha \beta C_{w_{y}} \lambda_{300}\right]$

Dryver and Chao (2007) proposed ratio estimator for population mean as,

$t_{d c}=\bar{w}_{y} \frac{\bar{X}_{w}}{\bar{w}_{x}}$

The bias and MSE of $t_{d c}$ to first order of approximation are,

$\operatorname{Bias}\left(t_{d c}\right)=\theta \bar{Y}_{w}\left(C_{w x}^{2}-C_{w_{y} w_{x}}\right)$

and

$$
\operatorname{MSE}\left(t_{d c}\right)=\theta \bar{Y}_{w}^{2}\left(C_{w y}^{2}+C_{w x}^{2}-2 C_{w_{y} w_{x}}\right)
$$

Following Dryver and Chao (2007), ratio estimator for general parameter $\tau_{(\alpha, \beta)}$, when $\left(\bar{X}_{w}\right)$ is unknown and $\left(\bar{Z}_{w}\right)$ is known, can be defined as,

$t_{d c-1}=\hat{\tau}_{(\alpha, \beta)}\left(\frac{{\overline{w_{x}}}^{\prime}}{\overline{w_{x}}}\right)\left(\begin{array}{l}\bar{w}_{z}^{\prime} \\ \overline{w_{z}}\end{array}\right)\left(\frac{\bar{Z}_{w}}{\overline{w_{z}}}\right)$,

The bias and MSE of $t_{d c-1}$ to first order of approximation are,

$$
\begin{aligned}
\operatorname{Bias}\left(t_{d c-1}\right)= & \tau_{(\alpha, \beta)}\left[\theta \left\{3 C_{w z}^{2}-2 \alpha C_{w_{y} w_{z}}-\beta \lambda_{201} C_{w_{z}}+\right.\right. \\
& \left.\frac{\alpha \beta}{2} C_{w_{y}} \lambda_{300}+\frac{\alpha(\alpha-1)}{2} C_{w y}^{2}+\frac{\beta(\beta-2)}{8} \lambda_{400}^{*}\right\} \\
& +\theta^{\prime}\left\{\alpha C_{w_{y} w_{z}}+\frac{\beta}{2} \lambda_{201} C_{w_{z}}-2 C_{w z}^{2}\right\}+ \\
& \left.\theta^{\prime \prime}\left\{C_{w x}^{2}+2 C_{w_{z} w_{x}}-\alpha C_{w_{y} w_{x}}-\frac{\beta}{2} \lambda_{210} C_{w_{x}}\right\}\right]
\end{aligned}
$$

and

$$
\begin{aligned}
\operatorname{MSE}\left(t_{d c-1}\right)= & \tau_{(\alpha, \beta)}^{2}\left[\theta \left\{\alpha^{2} C_{w y}^{2}+\frac{\beta^{2}}{4} \lambda_{400}^{*}-2 \beta \lambda_{201} C_{w_{z}}\right.\right. \\
& \left.+\frac{\alpha \beta}{2} C_{w_{y}} \lambda_{300}+4 C_{w z}^{2}-4 \alpha C_{w_{y} w_{z}}\right\} \\
& +\theta^{\prime}\left\{2 \alpha C_{w_{y} w_{z}}+\beta \lambda_{201} C_{w_{z}}-3 C_{w z}^{2}\right\}
\end{aligned}
$$

$$
+\theta^{\prime \prime}\left\{C_{w x}^{2}+4 C_{w_{z} w_{x}}-2 \alpha C_{w_{y} w_{x}}-\beta \lambda_{210} C_{w_{x}}\right\}
$$

Similarly, ratio estimator for general parameter $\tau_{(\alpha, \beta)}$, when both $\left(\bar{X}_{w}, \bar{Z}_{w}\right)$ are unknown, can be defined as,

$t_{d c-2}=\hat{\tau}_{(\alpha, \beta)}\left(\frac{\bar{w}_{x}^{\prime}}{\overline{w_{x}}}\right)\left(\begin{array}{l}\bar{w}_{z}^{\prime} \\ \overline{w_{z}}\end{array}\right)$

The bias and $M S E$ of $t_{d c-2}$, to first order of approximation are,

$$
\begin{aligned}
\operatorname{Bias}\left(t_{d c-2}\right)= & \tau_{(\alpha, \beta)}\left[\theta \left\{\frac{\alpha \beta}{2} C_{w_{y}} \lambda_{300}+\frac{\alpha(\alpha-1)}{2} C_{w y}^{2}\right.\right. \\
& \left.+\frac{\beta(\beta-2)}{8} \lambda_{400}^{*}\right\}+\theta^{\prime \prime}\left\{C_{w x}^{2}+C_{w z}^{2}+\right. \\
& C_{w_{x} w_{z}}-\frac{\beta}{2} \lambda_{210} C_{w_{x}}-\frac{\beta}{2} \lambda_{201} C_{w_{z}}- \\
& \left.\left.\alpha C_{w_{y} w_{x}}-\alpha C_{w_{y} w_{z}}\right\}\right]
\end{aligned}
$$

and

$$
\begin{aligned}
\operatorname{MSE}\left(t_{d c-2}\right)= & \tau_{(\alpha, \beta)}^{2}\left[\theta\left\{\alpha^{2} C_{w y}^{2}+\frac{\beta^{2}}{4} \lambda_{400}^{*}+\alpha \beta C_{w_{y}} \lambda_{300}\right\}\right. \\
& +\theta^{\prime \prime}\left\{C_{w x}^{2}+C_{w z}^{2}+2 C_{w_{x} w_{z}}-2 \alpha C_{w_{y} w_{x}}\right. \\
& \left.\left.-2 \alpha C_{w_{y} w_{z}}-\beta \lambda_{210} C_{w_{x}}-\beta \lambda_{201} C_{w z}\right\}\right]
\end{aligned}
$$

Also, ratio estimator for general parameter $\tau_{(\alpha, \beta)}$, when both $\left(\bar{X}_{w}, \bar{Z}_{w}\right)$ are known, can be defined as,

$t_{d c-3}=\hat{\tau}_{(\alpha, \beta)}\left(\frac{\bar{X}_{w}}{\bar{w}_{x}}\right)\left(\frac{\bar{Z}_{w}}{\bar{w}_{z}}\right)$

The bias and MSE of $t_{d c-3}$, to first order of approximation are,

$\operatorname{Bias}\left(t_{d c-3}\right)=\tau_{(\alpha, \beta)} \theta\left\{C_{w x}^{2}+C_{w z}^{2}-\alpha C_{w_{y} w_{x}}+\right.$

$\frac{\alpha(\alpha-1)}{2} C_{w y}^{2}+\frac{\beta(\beta-2)}{8} \lambda_{400}^{*}+\frac{\alpha \beta}{2} C_{w_{y}} \lambda_{300}$ 


$$
\left.+C_{w_{x} w_{z}}-\alpha C_{w_{y} w_{z}}-\frac{\beta}{2} \lambda_{210} C_{w_{x}}-\frac{\beta}{2} \lambda_{201} C_{w_{z}}\right\}
$$

and

$$
\begin{aligned}
\operatorname{MSE}\left(t_{d c-3}\right)= & \tau_{(\alpha, \beta)}^{2} \theta\left\{C_{w x}^{2}+C_{w z}^{2}+\alpha^{2} C_{w y}^{2}+\frac{\beta^{2}}{4} \lambda_{400}^{*}\right. \\
& +\alpha \beta C_{w_{y}} \lambda_{300}+2 C_{w_{x} w_{z}}-2 \alpha C_{w_{y} w_{x}} \\
& \left.-2 \alpha C_{w_{y} w_{z}}-\beta \lambda_{210} C_{w_{x}}-\beta \lambda_{201} C_{w z}\right\}
\end{aligned}
$$

\section{METHODOLOGY}

Estimator for general parameter when $\left(\bar{X}_{w}, S_{w_{x}}^{2}\right)$ are unknown and $\left(\bar{Z}_{w}, S_{w_{z}}^{2}\right)$ are known

Motivated by Shabbir and Gupta (2017), the following exponential ratio type estimator for the population parameter is proposed:

$$
\begin{aligned}
& t_{1}=\hat{\tau}_{(\alpha, \beta)} \exp \left\{\frac{\bar{w}_{x}^{\prime}-\bar{w}_{x}}{\bar{w}_{x}^{\prime}+(a-1) \bar{w}_{x}}\right\} \exp \left\{\frac{\bar{w}_{z}^{\prime}-\bar{w}_{z}}{\bar{w}_{z}^{\prime}+(c-1) \bar{w}_{z}}\right\} \\
& \exp \left\{\frac{\bar{Z}_{w}-\bar{w}_{z}}{\bar{Z}_{w}+(g-1) \bar{w}_{z}}\right\} \exp \left\{\frac{s_{w_{x}}^{2^{\prime}}-s_{w_{x}}^{2}}{s_{w_{x}}^{2^{2}}+(b-1) s_{w_{x}}^{2}}\right\} \\
& \exp \left\{\frac{s_{w_{z}}^{2^{\prime}}-s_{w_{z}}^{2}}{s_{w_{z}}^{2^{\prime}}+(d-1) s_{w_{z}}^{2}}\right\} \exp \left\{\frac{S_{w_{z}}^{2}-s_{w_{z}}^{2}}{S_{w_{z}}^{2}+(f-1) s_{w z}^{2}}\right\},
\end{aligned}
$$

where $a, b, c, d, g, f$ are suitably chosen constants. Rewriting equation (23) in terms of error terms and considering first order approximation, we have,

$$
\begin{aligned}
t_{1}= & \tau_{(\alpha, \beta)}\left\{1+\alpha e_{0}+\frac{\beta}{2} e_{3}+\frac{\alpha \beta}{2} e_{0} e_{3}+\frac{\alpha(\alpha-1)}{2} e_{0}^{2}+\right. \\
& \left.\frac{\beta(\beta-2)}{8} e_{3}^{2}\right\}\left\{1+\frac{e_{4}^{\prime}}{b}-\frac{e_{4}}{b}+\frac{e_{1}^{\prime}}{a}-\frac{e_{1}}{a}-\frac{e_{4}^{\prime 2}}{2 b^{2}}+\right. \\
& \frac{2 b-1}{2 b^{2}} e_{4}^{2}-\frac{e_{1}^{\prime 2}}{2 a^{2}}+\frac{2 a-1}{2 a^{2}} e_{1}^{2}+\frac{1-b}{b^{2}} e_{4} e_{4}^{\prime}+ \\
& \left.\frac{1-a}{a^{2}} e_{1} e_{1}^{\prime}+\frac{e_{1}^{\prime} e_{4}^{\prime}}{a b}-\frac{e_{1}^{\prime} e_{4}}{a b}-\frac{e_{1} e_{4}^{\prime}}{a b}+\frac{e_{1} e_{4}}{a b}\right\} \\
\} & \left\{1+\frac{e_{5}^{\prime}}{d}-\frac{e_{5}}{d}+\frac{e_{2}^{\prime}}{c}-\frac{e_{2}}{c}-\frac{e_{5}^{\prime 2}}{2 d^{2}}+\frac{2 d-1}{2 d^{2}} e_{5}^{2}+\right.
\end{aligned}
$$

$$
\begin{aligned}
& \frac{2 c-1}{2 c^{2}} e_{2}^{2}-\frac{e_{2}^{\prime 2}}{2 c^{2}}+\frac{1-d}{d^{2}} e_{5} e_{5}^{\prime}+\frac{1-c}{c^{2}} e_{2} e_{2}^{\prime}+ \\
& \left.\frac{e_{2}^{\prime} e_{5}^{\prime}}{c d}-\frac{e_{2}^{\prime} e_{5}}{c d}-\frac{e_{2} e_{5}^{\prime}}{c d}+\frac{e_{2} e_{5}}{c d}\right\}\left\{1-\frac{e_{5}}{f}-\frac{e_{2}}{g}\right. \\
& \left.+\frac{2 f-1}{2 f^{2}} e_{5}^{2}+\frac{2 g-1}{2 g^{2}} e_{2}^{2}+\frac{e_{2} e_{5}}{g f}\right\}
\end{aligned}
$$

Multiplying out the terms on right hand side, we have,

$$
\begin{aligned}
& t_{1}=\tau_{(\alpha, \beta)}\left[1+\frac{e_{5}^{\prime}}{d}-\frac{e_{5}}{d}+\frac{e_{2}^{\prime}}{c}-\frac{e_{2}}{c}+\frac{e_{4}^{\prime}}{b}-\frac{e_{4}}{b}+\frac{e_{1}^{\prime}}{a}-\frac{e_{1}}{a}\right. \\
& +\alpha e_{0}+\frac{\beta}{2} e_{3}-\frac{e_{5}}{f}-\frac{e_{2}}{g}+\left(\frac{2 d-1}{2 d^{2}}+\frac{1}{d f}\right)\left(e_{5}^{2}-e_{5}^{2}\right) \\
& +\left(\frac{2 c-1}{2 c^{2}}+\frac{1}{c g}\right)\left(e_{2}^{2}-e_{2}^{\prime 2}\right)+\left(\frac{2 f-1}{2 f^{2}}\right) e_{5}^{2}+ \\
& \frac{2 b-1}{2 b^{2}}\left(e_{4}^{2}-e_{4}^{\prime 2}\right)+\left(\frac{1}{c d}+\frac{1}{c f}+\frac{1}{d g}\right)\left(e_{2} e_{5}-e_{2} e_{5}^{\prime}\right)+ \\
& \left(\frac{2 g-1}{2 g^{2}}\right) e_{2}^{2}+\frac{1}{g f} e_{2} e_{5}+\left(\frac{1}{b d}+\frac{1}{b f}\right)\left(e_{4} e_{5}-e_{4} e_{5}^{\prime}\right) \\
& +\frac{2 a-1}{2 a^{2}}\left(e_{1}^{2}-e_{1}^{\prime 2}\right)+\left(\frac{1}{b c}+\frac{1}{b g}\right)\left(e_{2} e_{4}-e_{2} e_{4}^{\prime}\right)+ \\
& \frac{\alpha(\alpha-1)}{2} e_{0}^{2}+\frac{1}{a b}\left(e_{1} e_{4}-e_{1} e_{4}^{\prime}\right) \\
& +\left(\frac{1}{a d}+\frac{1}{a f}\right)\left(e_{1} e_{5}-e_{1} e_{5}^{\prime}\right)-\frac{\alpha}{g} e_{0} e_{2}-\frac{\alpha}{f} e_{0} e_{5}+ \\
& +\left(\frac{1}{a c}+\frac{1}{a g}\right)\left(e_{1} e_{2}-e_{1} e_{2}^{\prime}\right)-\frac{\beta}{2 f} e_{3} e_{5} \\
& +\frac{\beta(\beta-2)}{8} e_{3}^{2}-\frac{\alpha}{d}\left(e_{0} e_{5}-e_{0} e_{5}^{\prime}\right)-\frac{\alpha}{c}\left(e_{0} e_{2}-e_{0} e_{2}^{\prime}\right) \\
& -\frac{\alpha}{b}\left(e_{0} e_{4}-e_{0} e_{4}^{\prime}\right)-\frac{\beta}{2 g} e_{3} e_{2}-\frac{\alpha}{a}\left(e_{0} e_{1}-e_{0} e_{1}^{\prime}\right) \\
& -\frac{\beta}{2 d}\left(e_{3} e_{5}-e_{3} e_{5}^{\prime}\right)-\frac{\beta}{2 c}\left(e_{3} e_{2}-e_{3} e_{2}^{\prime}\right)- \\
& \left.\frac{\beta}{2 b}\left(e_{3} e_{4}-e_{3} e_{4}^{\prime}\right)-\frac{\beta}{2 a}\left(e_{3} e_{1}-e_{3} e_{1}^{\prime}\right)+\frac{\alpha \beta}{2} e_{0} e_{3}\right]
\end{aligned}
$$


From equation (25), we get the bias of proposed estimator $t_{1}$ as,

$$
\begin{aligned}
& \operatorname{Bias}\left(t_{1}\right)=\tau_{(\alpha, \beta)}\left[\theta \left\{\frac{w_{6}\left(2-w_{6}\right)}{2} \lambda_{004}^{*}+\frac{w_{5}\left(2-w_{5}\right)}{2} C_{w_{z}}^{2}\right.\right. \\
& +w_{5} w_{6} \lambda_{003} C_{w_{z}}+\frac{\alpha \beta}{2} C_{w_{y}} \lambda_{300}+\frac{\alpha(\alpha-1)}{2} C_{w_{y}}^{2}+ \\
& \frac{\beta(\beta-2)}{8} \lambda_{400}^{*}-\alpha w_{6} \lambda_{102} C_{w_{y}}-\alpha w_{5} C_{w_{y} w_{z}}-\frac{\beta}{2} w_{6} \lambda_{202}^{*} \\
& \left.-\frac{\beta}{2} w_{5} \lambda_{201} C_{w_{z}}\right\}+\theta^{\prime \prime}\left\{\frac{w_{1}\left(2-w_{1}\right)}{2} C_{w_{x}}^{2}+\right. \\
& \left(w_{3} w_{4}+w_{3} w_{6}+w_{4} w_{5}\right) \lambda_{003} C_{w_{z}}+\left(\frac{w_{4}\left(2-w_{4}\right)}{2}+\right. \\
& \left.w_{4} w_{6}\right) \lambda_{004}^{*}+\left(w_{1} w_{4}+w_{1} w_{6}\right) \lambda_{012} C_{w_{x}}-\alpha w_{2} \lambda_{120} C_{w_{y}} \\
& \operatorname{MSE}\left(t_{1}\right)=\tau_{(\alpha, \beta)}^{2}\left[\theta \left\{\alpha^{2} C_{w_{y}}^{2}+w_{5}^{2} C_{w_{z}}^{2}+\frac{\beta^{2}}{4} \lambda_{400}^{*}+2 w_{5} w_{6} \lambda_{003} C_{w_{z}}-2 \alpha w_{6} \lambda_{102} C_{w_{y}}+w_{6}^{2} \lambda_{004}^{*}\right.\right. \\
& \left.-2 \alpha w_{5} C_{w_{y} w_{z}}-\beta w_{6} \lambda_{202}^{*}-\beta w_{5} \lambda_{201} C_{w_{z}}+\alpha \beta C_{w_{y}} \lambda_{300}\right\}+\theta^{\prime \prime}\left\{w_{1}^{2} C_{w_{x}}^{2}+w_{2}^{2} \lambda_{040}^{*}\right. \\
& +C_{w_{z}}^{2}\left(w_{3}^{2}+2 w_{3} w_{5}\right)+\lambda_{004}^{*}\left(w_{4}^{2}+2 w_{4} w_{6}\right)+2 \lambda_{003} C_{w_{z}}\left(w_{3} w_{4}+w_{3} w_{6}+w_{4} w_{5}\right) \\
& +2 \lambda_{022}^{*}\left(w_{2} w_{4}+w_{2} w_{6}\right)+2 \lambda_{021} C_{w_{z}}\left(w_{2} w_{3}+w_{2} w_{5}\right)+2 C_{w_{x} w_{z}}\left(w_{1} w_{3}+w_{1} w_{5}\right) \\
& +2 \lambda_{012} C_{w_{x}}\left(w_{1} w_{4}+w_{1} w_{6}\right)+2 w_{1} w_{2} \lambda_{030} C_{w_{x}}-2 \alpha w_{4} \lambda_{102} C_{w_{y}}-2 \alpha w_{1} C_{w_{y} w_{x}} \\
& -2 \alpha w_{3} C_{w_{v} w_{z}}-2 \alpha w_{2} \lambda_{120} C_{w_{y}}-\beta w_{4} \lambda_{202}^{*}-\beta w_{3} \lambda_{201} C_{w_{z}}-\beta w_{2} \lambda_{220}^{*} \\
& \left.\left.-\beta w_{1} \lambda_{210} C_{w_{x}}\right\}\right]
\end{aligned}
$$

$$
\begin{aligned}
& +\frac{w_{2}\left(2-w_{2}\right)}{2} \lambda_{040}^{*}+\left(w_{2} w_{4}+w_{2} w_{6}\right) \lambda_{022}^{*}+ \\
& w_{1} w_{2} \lambda_{030} C_{w_{x}}-\frac{\beta}{2} w_{1} \lambda_{210} C_{w_{x}}+\left(\frac{w_{3}\left(2-w_{3}\right)}{2}+w_{3} w_{5}\right) C_{w_{z}}^{2} \\
& +\left(w_{2} w_{3}+w_{2} w_{5}\right) \lambda_{021} C_{w_{z}}-\frac{\beta}{2} w_{3} \lambda_{201} C_{w_{z}} \\
& +\left(w_{1} w_{3}+w_{1} w_{5}\right) C_{w_{x} w_{z}}-\alpha w_{4} \lambda_{102} C_{w_{y}}-\alpha w_{3} C_{w_{y} w_{z}} \\
& \left.\left.-\alpha w_{1} C_{w_{y} w_{x}}-\frac{\beta}{2} w_{4} \lambda_{202}^{*}-\frac{\beta}{2} w_{2} \lambda_{220}^{*}\right\}\right]
\end{aligned}
$$

where $\quad w_{1}=\frac{1}{a}, w_{2}=\frac{1}{b}, w_{3}=\frac{1}{c}, w_{4}=\frac{1}{d}, w_{5}=\frac{1}{g}$, $w_{6}=\frac{1}{f}$. The mean square error of the proposed estimator $t_{1}$, to the first degree of approximation is

We get minimum mean square error of $t_{1}$ by substituting of $w_{i} \forall i=1,2, \ldots, 6$ are obtained by solving the optimum values of $w_{i} \forall i=1,2, \ldots, 6$. Optimum values $\quad A X=B$

where

$$
\begin{aligned}
A & =\left[\begin{array}{cccccc}
2 C_{w_{x}} & 2 \lambda_{030} & 2 \rho_{w_{x} w_{z}} C_{w_{z}} & 2 \lambda_{012} & 2 \rho_{w_{x} w_{z}} C_{w_{z}} & 2 \lambda_{012} \\
2 \lambda_{030} C_{w_{x}} & 2 \lambda_{040}^{*} & 2 \lambda_{021} C_{w_{z}} & 2 \lambda_{022}^{*} & 2 \lambda_{021} C_{w_{z}} & 2 \lambda_{022}^{*} \\
2 \rho_{w_{x} w_{z}} C_{w_{x}} & 2 \lambda_{021} & 2 C_{w_{z}} & 2 \lambda_{003} & 2 C_{w_{z}} & 2 \lambda_{003} \\
2 \lambda_{012} C_{w_{x}} & 2 \lambda_{022}^{*} & 2 \lambda_{003} C_{w_{z}} & 2 \lambda_{004}^{*} & 2 \lambda_{003} C_{w_{z}} & 2 \lambda_{004}^{*} \\
2 \rho_{w_{x} w_{z}} C_{w_{x}} \theta^{\prime \prime} & 2 \lambda_{021} \theta^{\prime \prime} & 2 C_{w_{z}} \theta^{\prime \prime} & 2 \lambda_{003} \theta^{\prime \prime} & 2 C_{w_{z}} \theta & 2 \lambda_{003} \theta \\
2 \lambda_{012} C_{w_{x}} \theta^{\prime \prime} & 2 \lambda_{022}^{*} \theta^{\prime \prime} & 2 \lambda_{003} C_{w_{z}} \theta^{\prime \prime} & 2 \lambda_{004}^{*} \theta^{\prime \prime} & 2 \lambda_{003} C_{w_{z}} \theta & 2 \lambda_{004}^{*} \theta
\end{array}\right], \\
X & =\left[\begin{array}{c}
w_{1} \\
w_{2} \\
w_{3} \\
w_{4} \\
w_{5} \\
w_{6}
\end{array}\right], B=\left[\begin{array}{c}
2 \alpha \rho_{w_{x} w_{y}} C_{w y}+\beta \lambda_{210} \\
2 \alpha \lambda_{120} C_{w_{y}}+\beta \lambda_{220}^{*} \\
2 \alpha \rho_{w_{z} w_{y}} C_{w y}+\beta \lambda_{201} \\
2 \alpha \lambda_{102} C_{w_{y}}+\beta \lambda_{202}^{*} \\
2 \alpha \rho_{w_{z} w_{y}} C_{w y} \theta+\beta \lambda_{201} \theta \\
2 \alpha \lambda_{102} C_{w_{y}} \theta+\beta \lambda_{202}^{*} \theta
\end{array}\right]
\end{aligned}
$$


The exponential ratio type estimator for population mean $t_{1 m}$ can be obtained by substituting $\alpha=1, \beta=0$ in equation (23). The bias and $M S E$ of $t_{1 m}$ can be obtained by putting $\alpha=1, \beta=0$ in equations (26) and
(27). Similarly, exponential ratio type estimator for population variance $\left(t_{1 v}\right)$ and coefficient of variation $\left(t_{1 c}\right)$ can be obtained by putting $\alpha=0, \beta=2$ and $\alpha=-1, \beta=1$ respectively in equation (23).

Estimator for general parameter when all $\left(\bar{X}_{w}, S_{w_{x}}^{2}\right)$ and $\left(\bar{Z}_{w}, S_{w_{z}}^{2}\right)$ are unknown

When $\bar{X}_{w}, S_{w_{x}}^{2}$ and $\bar{Z}_{w}, S_{w_{z}}^{2}$ all are unknown, the following estimator for general parameter is proposed:

$$
t_{2}=\hat{\tau}_{(\alpha, \beta)} \exp \left\{\frac{\bar{w}_{x}^{\prime}-\bar{w}_{x}}{\overline{\bar{w}_{x}^{\prime}}+(a-1) \bar{w}_{x}}\right\} \exp \left\{\frac{\bar{w}_{z}^{\prime}-\bar{w}_{z}}{\overline{\bar{w}_{z}^{\prime}}+(c-1) \bar{w}_{z}}\right\} \exp \left\{\frac{s_{w_{x}}^{2^{\prime}}-s_{w_{x}}^{2}}{s_{w_{x}}^{2^{\prime}}+(b-1) s_{w_{x}}^{2}}\right\} \exp \left\{\frac{s_{w_{z}}^{2^{\prime}}-s_{w_{z}}^{2}}{s_{w_{z}}^{2^{\prime}}+(d-1) s_{w_{z}}^{2}}\right\},
$$

where $a, b, c, d$ are suitably chosen constants. Rewriting equation (29) in terms of error terms and considering first order approximation, we have,

$$
\begin{aligned}
t_{2}= & \tau_{(\alpha, \beta)}\left\{1+\alpha e_{0}+\frac{\beta}{2} e_{3}+\frac{\alpha \beta}{2} e_{0} e_{3}+\frac{\alpha(\alpha-1)}{2} e_{0}^{2}+\frac{\beta(\beta-2)}{8} e_{3}^{2}\right\}\left\{1+\frac{e_{4}^{\prime}}{b}-\frac{e_{4}}{b}+\frac{e_{1}^{\prime}}{a}-\frac{e_{1}}{a}-\frac{e_{4}^{\prime 2}}{2 b^{2}}+\frac{2 b-1}{2 b^{2}} e_{4}^{2}-\right. \\
& \left.\frac{e_{1}^{\prime 2}}{2 a^{2}}+\frac{2 a-1}{2 a^{2}} e_{1}^{2}+\frac{1-b}{b^{2}} e_{4} e_{4}^{\prime}+\frac{e_{1}^{\prime} e_{4}^{\prime}}{a b}+\frac{e_{1} e_{4}}{a b}+\frac{1-a}{a^{2}} e_{1} e_{1}^{\prime}-\frac{e_{1}^{\prime} e_{4}}{a b}-\frac{e_{1} e_{4}^{\prime}}{a b}\right\}\left\{1+\frac{e_{5}^{\prime}}{d}-\frac{e_{5}}{d}+\frac{e_{2}^{\prime}}{c}-\frac{e_{2}}{c}-\frac{e_{5}^{\prime 2}}{2 d^{2}}+\right. \\
& \left.\frac{2 d-1}{2 d^{2}} e_{5}^{2}+\frac{1-d}{d^{2}} e_{5} e_{5}^{\prime}+\frac{1-c}{c^{2}} e_{2} e_{2}^{\prime}-\frac{e_{2}^{\prime 2}}{2 c^{2}}+\frac{2 c-1}{2 c^{2}} e_{2}^{2}+\frac{e_{2}^{\prime} e_{5}^{\prime}}{c d}-\frac{e_{2}^{\prime} e_{5}}{c d}-\frac{e_{2} e_{5}^{\prime}}{c d}+\frac{e_{2} e_{5}}{c d}\right\}
\end{aligned}
$$

Multiplying out the terms on right hand side, we have,

$$
\begin{aligned}
t_{2}= & \tau_{(\alpha, \beta)}\left[1+\frac{e_{5}^{\prime}}{d}-\frac{e_{5}}{d}+\frac{e_{2}^{\prime}}{c}-\frac{e_{2}}{c}+\frac{e_{4}^{\prime}}{b}-\frac{e_{4}}{b}+\frac{e_{1}^{\prime}}{a}-\frac{e_{1}}{a}+\alpha e_{0}+\frac{\alpha(\alpha-1)}{2} e_{0}^{2}+\frac{\beta}{2} e_{3}+\frac{\beta(\beta-2)}{8} e_{3}^{2}+\right. \\
& \frac{2 a-1}{2 a^{2}}\left(e_{1}^{2}-e_{1}^{\prime 2}\right)+\frac{2 b-1}{2 b^{2}}\left(e_{4}^{2}-e_{4}^{\prime 2}\right)+\frac{\alpha \beta}{2} e_{0} e_{3}+\frac{2 c-1}{2 c^{2}}\left(e_{2}^{2}-e_{2}^{\prime 2}\right)+\frac{2 d-1}{2 d^{2}}\left(e_{5}^{2}-e_{5}^{\prime 2}\right)+\frac{1}{c d}\left(e_{2} e_{5}-e_{2} e_{5}^{\prime}\right)+ \\
& \frac{1}{b d}\left(e_{4} e_{5}-e_{4} e_{5}^{\prime}\right)+\frac{1}{b c}\left(e_{2} e_{4}-e_{2} e_{4}^{\prime}\right)+\frac{1}{a d}\left(e_{1} e_{5}-e_{1} e_{5}^{\prime}\right)+\frac{1}{a c}\left(e_{1} e_{2}-e_{1} e_{2}^{\prime}\right)+\frac{1}{a b}\left(e_{1} e_{4}-e_{1} e_{4}^{\prime}\right) \\
& -\frac{\alpha}{d}\left(e_{0} e_{5}-e_{0} e_{5}^{\prime}\right)-\frac{\alpha}{c}\left(e_{0} e_{2}-e_{0} e_{2}^{\prime}\right)-\frac{\beta}{2 a}\left(e_{3} e_{1}-e_{3} e_{1}^{\prime}\right)-\frac{\beta}{2 b}\left(e_{3} e_{4}-e_{3} e_{4}^{\prime}\right) \\
& \left.-\frac{\alpha}{a}\left(e_{0} e_{1}-e_{0} e_{1}^{\prime}\right)-\frac{\alpha}{b}\left(e_{0} e_{4}-e_{0} e_{4}^{\prime}\right)-\frac{\beta}{2 c}\left(e_{3} e_{2}-e_{3} e_{2}^{\prime}\right)-\frac{\beta}{2 d}\left(e_{3} e_{5}-e_{3} e_{5}^{\prime}\right)\right]
\end{aligned}
$$


From equation (31), we get the bias of proposed estimator $t_{2}$ as,

$$
\begin{aligned}
\operatorname{Bias}\left(t_{2}\right)= & \tau_{(\alpha, \beta)}\left[\theta\left\{\frac{\alpha(\alpha-1)}{2} C_{w_{y}}^{2}+\frac{\beta(\beta-2)}{8} \lambda_{400}^{*}+\frac{\alpha \beta}{2} C_{w_{y}} \lambda_{300}\right\}+\theta^{\prime \prime}\left\{w_{1} w_{3} C_{w_{x} w_{z}}+\frac{w_{4}\left(2-w_{4}\right)}{2} \lambda_{004}^{*}+\right.\right. \\
& \frac{w_{1}\left(2-w_{1}\right)}{2} C_{w_{x}}^{2}+\frac{w_{3}\left(2-w_{3}\right)}{2} C_{w_{z}}^{2}+w_{3} w_{4} \lambda_{003} C_{w_{z}}+w_{1} w_{4} \lambda_{012} C_{w_{x}}+w_{1} w_{2} \lambda_{030} C_{w_{x}}- \\
& -\alpha w_{1} C_{w_{y} w_{x}}-\alpha w_{2} \lambda_{120} C_{w_{y}}-\alpha w_{3} C_{w_{y} w_{z}}+\frac{w_{2}\left(2-w_{2}\right)}{2} \lambda_{040}^{*}+w_{2} w_{3} \lambda_{021} C_{w_{z}}+w_{2} w_{4} \lambda_{022}^{*}- \\
& \left.\left.\frac{\beta}{2} w_{4} \lambda_{202}^{*}-\frac{\beta}{2} w_{2} \lambda_{220}^{*}-\frac{\beta}{2} w_{3} \lambda_{201} C_{w_{z}}-\alpha w_{4} \lambda_{102} C_{w_{y}}-\frac{\beta}{2} w_{1} \lambda_{210} C_{w_{x}}\right\}\right]
\end{aligned}
$$

The MSE of proposed estimator $t_{2}$, to the first degree of approximation is obtained as,

$$
\begin{aligned}
& \operatorname{MSE}\left(t_{2}\right)=\tau_{(\alpha, \beta)}^{2}\left[\theta\left\{\alpha^{2} C_{w_{y}}^{2}+\frac{\beta^{2}}{4} \lambda_{400}^{*}+\alpha \beta C_{w_{y}} \lambda_{300}\right\}+\theta^{\prime \prime}\left\{w_{4}^{2} \lambda_{004}^{*}+w_{3}^{2} C_{w_{z}}^{2}+2 w_{2} w_{4} \lambda_{022}^{*}+w_{1}^{2} C_{w_{x}}^{2}+\right.\right. \\
& 2 w_{3} w_{4} \lambda_{003} C_{w_{z}}-2 \alpha w_{1} C_{w_{y} w_{x}}+2 w_{1} w_{4} \lambda_{012} C_{w_{x}}+2 w_{1} w_{2} \lambda_{030} C_{w_{x}}+w_{2}^{2} \lambda_{040}^{*}+2 w_{2} w_{3} \lambda_{021} C_{w_{z}}+ \\
& 2 w_{1} w_{3} C_{w_{x} w_{z}}-2 \alpha w_{4} \lambda_{102} C_{w_{y}}-2 \alpha w_{2} \lambda_{120} C_{w_{y}}-2 \alpha w_{3} C_{w_{y} w_{z}}-\beta w_{1} \lambda_{210} C_{w_{x}}-\beta w_{2} \lambda_{220}^{*}- \\
&\left.\left.\beta w_{3} \lambda_{201} C_{w_{z}}-\beta w_{4} \lambda_{202}^{*}\right\}\right]
\end{aligned}
$$

The exponential ratio type estimator for population mean $t_{2 m}$ can be obtained by substituting $\alpha=1, \beta=0$ in equation (29). The bias and $M S E$ of $t_{2 m}$ can be obtained by putting $\alpha=1, \beta=0$ in equations (32) and (33). Similarly, exponential ratio type estimator for population variance $\left(t_{2 v}\right)$ and coefficient of variation $\left(t_{2 c}\right)$ can be obtained by putting $\alpha=0, \beta=2$ and $\alpha=-1, \beta=1$ respectively in equation (29).

Estimator for general parameter when all $\left(\bar{X}_{w}, S_{w_{x}}^{2}\right)$ and $\left(\bar{Z}_{w}, S_{w_{z}}^{2}\right)$ are known

When $\bar{X}_{w}, S_{w_{x}}^{2}$ and $\bar{Z}_{w}, S_{w_{z}}^{2}$ all are known, we propose the following estimator for general parameter:

$$
\begin{aligned}
t_{3}= & \hat{\tau}_{(\alpha, \beta)} \exp \left\{\frac{\bar{X}_{w}-\bar{w}_{x}}{\bar{X}_{w}+(a-1) \bar{w}_{x}}\right\} \exp \left\{\frac{\bar{Z}_{w}-\bar{w}_{z}}{\bar{Z}_{w}+(c-1) \bar{w}_{z}}\right\} \\
& \exp \left\{\frac{S_{w_{x}}^{2}-s_{w_{x}}^{2}}{S_{w_{x}}^{2}+(b-1) s_{w_{x}}^{2}}\right\} \exp \left\{\frac{S_{w_{z}}^{2}-s_{w_{z}}^{2}}{S_{w_{z}}^{2}+(d-1) s_{w_{z}}^{2}}\right\}
\end{aligned}
$$

where $a, b, c, d$ are suitably chosen constants. Rewriting equation (34) in terms of error terms and considering first order approximation, we have,

$$
\begin{aligned}
t_{3}= & \tau_{(\alpha, \beta)}\left\{1+\alpha e_{0}+\frac{\beta}{2} e_{3}+\frac{\alpha \beta}{2} e_{0} e_{3}+\frac{\alpha(\alpha-1)}{2} e_{0}^{2}+\right. \\
& \left.\frac{\beta(\beta-2)}{8} e_{3}^{2}\right\}\left\{1-\frac{e_{4}}{b}-\frac{e_{1}}{a}+\frac{2 b-1}{2 b^{2}} e_{4}^{2}+\frac{2 a-1}{2 a^{2}} e_{1}^{2}+\frac{e_{1} e_{4}}{a b}\right\} \\
& \left\{1-\frac{e_{5}}{d}-\frac{e_{2}}{c}+\frac{2 d-1}{2 d^{2}} e_{5}^{2}+\frac{2 c-1}{2 c^{2}} e_{2}^{2}+\frac{e_{2} e_{5}}{c d}\right\}
\end{aligned}
$$

Multiplying out the terms on right hand side, we have,

$$
\begin{aligned}
t_{3} & =\tau_{(\alpha, \beta)}\left[1-\frac{e_{5}}{d}-\frac{e_{2}}{c}-\frac{e_{4}}{b}-\frac{e_{1}}{a}+\alpha e_{0}+\frac{\beta}{2} e_{3}-\frac{\alpha}{d} e_{0} e_{5}-\frac{\alpha}{c} e_{0} e_{2}\right. \\
& +\frac{2 d-1}{2 d^{2}} e_{5}^{2}-\frac{\alpha}{b} e_{0} e_{4}-\frac{\alpha}{a} e_{0} e_{1}+\frac{2 c-1}{2 c^{2}} e_{2}^{2}+\frac{2 b-1}{2 b^{2}} e_{4}^{2}+\frac{2 a-1}{2 a^{2}} e_{1}^{2} \\
& +\frac{\alpha \beta}{2} e_{0} e_{3}+\frac{1}{c d} e_{2} e_{5}+\frac{1}{b d} e_{4} e_{5}+\frac{\alpha(\alpha-1)}{2} e_{0}^{2}+\frac{1}{b c} e_{2} e_{4}+\frac{1}{a d} e_{1} e_{5} \\
& +\frac{1}{a c} e_{1} e_{2}+\frac{1}{a b} e_{1} e_{4}-\frac{\beta}{2 a} e_{3} e_{1}+\frac{\beta(\beta-2)}{8} e_{3}^{2}-\frac{\beta}{2 c} e_{3} e_{2} \\
& \left.-\frac{\beta}{2 b} e_{3} e_{4}-\frac{\beta}{2 d} e_{3} e_{5}\right]
\end{aligned}
$$

From equation (36), we get the bias of proposed estimator $t_{3}$ as, 


$$
\begin{aligned}
\operatorname{Bias}\left(t_{3}\right)= & \tau_{(\alpha, \beta)} \theta\left[\frac{w_{1}\left(2-w_{1}\right)}{2} C_{w_{x}}^{2}+\frac{w_{2}\left(2-w_{2}\right)}{2} \lambda_{040}^{*}+\frac{w_{3}\left(2-w_{3}\right)}{2} C_{w_{z}}^{2}+w_{1} w_{3} C_{w_{x} w_{z}}\right. \\
& +\frac{w_{4}\left(2-w_{4}\right)}{2} \lambda_{004}^{*}+\frac{\alpha(\alpha-1)}{2} C_{w_{y}}^{2}+w_{1} w_{4} \lambda_{012} C_{w_{x}}+w_{2} w_{4} \lambda_{022}^{*}-\alpha w_{1} C_{w_{y} w_{x}} \\
& +\frac{\beta(\beta-2)}{8} \lambda_{400}^{*}+\frac{\alpha \beta}{2} C_{w_{y}} \lambda_{300}+w_{2} w_{3} \lambda_{021} C_{w_{z}}+w_{3} w_{4} \lambda_{003} C_{w_{z}}-\alpha w_{3} C_{w_{y} w_{z}} \\
& +w_{1} w_{2} \lambda_{030} C_{w_{x}}-\alpha w_{2} \lambda_{120} C_{w_{y}}-\alpha w_{4} \lambda_{102} C_{w_{y}}-\frac{\beta}{2} w_{2} \lambda_{220}^{*}-\frac{\beta}{2} w_{1} \lambda_{210} C_{w_{x}} \\
& \left.-\frac{\beta}{2} w_{4} \lambda_{202}^{*}-\frac{\beta}{2} w_{3} \lambda_{201} C_{w_{z}}\right]
\end{aligned}
$$

The mean square error of the proposed estimator $t_{3}$, to the first degree of approximation is obtained as

$$
\begin{aligned}
\operatorname{MSE}\left(t_{3}\right)= & \tau_{(\alpha, \beta)}^{2} \theta\left[\alpha^{2} C_{w_{y}}^{2}+\frac{\beta^{2}}{4} \lambda_{400}^{*}+\alpha \beta C_{w_{y}} \lambda_{300}+w_{2}^{2} \lambda_{040}^{*}+w_{1}^{2} C_{w_{x}}^{2}+w_{3}^{2} C_{w_{z}}^{2}+w_{4}^{2} \lambda_{004}^{*}\right. \\
& +2 w_{3} w_{4} \lambda_{003} C_{w_{z}}+2 w_{2} w_{4} \lambda_{022}^{*}+2 w_{1} w_{4} \lambda_{012} C_{w_{x}}-2 \alpha w_{1} C_{w_{y} w_{x}}-2 \alpha w_{3} C_{w_{y} w_{z}} \\
& +2 w_{1} w_{2} \lambda_{030} C_{w_{x}}+2 w_{1} w_{3} C_{w_{x} w_{z}}-2 \alpha w_{2} \lambda_{120} C_{w_{y}}-\beta w_{1} \lambda_{210} C_{w_{x}}-\beta w_{2} \lambda_{220}^{*} \\
& \left.+2 w_{2} w_{3} \lambda_{021} C_{w_{z}}-2 \alpha w_{4} \lambda_{102} C_{w_{y}}-\beta w_{3} \lambda_{201} C_{w_{z}}-\beta w_{4} \lambda_{202}^{*}\right]
\end{aligned}
$$

Optimum values of $w_{i} \forall i=1,2, \ldots, 4$ for $t_{2}$ and $t_{3}$ can be obtained by solving the following system of equations:

$$
\left[\begin{array}{cccc}
2 C_{w_{x}} & 2 \lambda_{030} & 2 \rho_{w_{x} w_{z}} C_{w_{z}} & 2 \lambda_{012} \\
2 \lambda_{030} C_{w_{x}} & 2 \lambda_{040}^{*} & 2 \lambda_{021} C_{w_{z}} & 2 \lambda_{022}^{*} \\
2 \rho_{w_{x} w_{z}} C_{w_{x}} & 2 \lambda_{021} & 2 C_{w_{z}} & 2 \lambda_{003} \\
2 \lambda_{012} C_{w_{x}} & 2 \lambda_{022}^{*} & 2 \lambda_{003} C_{w_{z}} & 2 \lambda_{004}^{*}
\end{array}\right]\left[\begin{array}{c}
w_{1} \\
w_{2} \\
w_{3} \\
w_{4}
\end{array}\right]=\left[\begin{array}{c}
2 \alpha \rho_{w_{x} w_{y}} C_{w y}+\beta \lambda_{210} \\
2 \alpha \lambda_{120} C_{w_{y}}+\beta \lambda_{220}^{*} \\
2 \alpha \rho_{w_{z} w_{y}} C_{w y}+\beta \lambda_{201} \\
2 \alpha \lambda_{102} C_{w_{y}}+\beta \lambda_{202}^{*}
\end{array}\right]
$$

The exponential ratio type estimator for population mean $t_{3 m}$ can be obtained by substituting $\alpha=1, \beta=0$ in equation (34). The bias and MSE of $t_{3 m}$ can be obtained by putting $\alpha=1, \beta=0$ in equations (37) and (38). Similarly, the exponential ratio type estimator for population variance $\left(t_{3 v}\right)$ and coefficient of variation $\left(t_{3 c}\right)$ can be obtained by putting $\alpha=0, \beta=2$ and $\alpha=-1, \beta=1$ respectively in equation (34).

\section{Efficiency comparison}

Comparison of the proposed estimators with other estimators are considered here.

From equations (10) and (27), $\operatorname{MSE}\left(t_{1}\right)<\operatorname{MSE}\left(t_{0}\right)$ if

$$
\begin{aligned}
& \theta\left\{w_{6}^{2} \lambda_{004}^{*}+w_{5}^{2} C_{w_{z}}^{2}+2 w_{5} w_{6} \lambda_{003} C_{w_{z}}-2 \alpha w_{6} \lambda_{102} C_{w_{y}}-2 \alpha w_{5} C_{w_{y} w_{z}}-\beta w_{5} \lambda_{201} C_{w_{z}}-\beta w_{6} \lambda_{202}^{*}\right. \\
& \}+\theta^{\prime \prime}\left\{\lambda_{004}^{*}\left(w_{4}^{2}+2 w_{4} w_{6}\right)+2 \lambda_{003} C_{w_{z}}\left(w_{3} w_{4}+w_{3} w_{6}+w_{4} w_{5}\right)+C_{w_{z}}^{2}\left(w_{3}^{2}+2 w_{3} w_{5}\right)+w_{1}^{2} C_{w_{x}}^{2}\right. \\
& +2 w_{1} w_{2} \lambda_{030} C_{w_{x}}+w_{2}^{2} \lambda_{040}^{*}+2 \lambda_{012} C_{w_{x}}\left(w_{1} w_{4}+w_{1} w_{6}\right)+2\left(w_{2} w_{4}+w_{2} w_{6}\right) \lambda_{022}^{*}-\beta w_{4} \lambda_{202}^{*} \\
& +2\left(w_{2} w_{3}+w_{2} w_{5}\right) \lambda_{021} C_{w_{z}}+2\left(w_{1} w_{3}+w_{1} w_{5}\right) C_{w_{x} w_{z}}-2 \alpha w_{2} \lambda_{120} C_{w_{y}}-2 \alpha w_{4} \lambda_{102} C_{w_{y}} \\
& \left.-2 \alpha w_{3} C_{w_{y} w_{z}}-2 \alpha w_{1} C_{w_{y} w_{x}}-\beta w_{3} \lambda_{201} C_{w_{z}}-\beta w_{2} \lambda_{220}^{*}-\beta w_{1} \lambda_{210} C_{w_{x}}\right\}<0
\end{aligned}
$$


From equations (16) and (27), $\operatorname{MSE}\left(t_{1}\right)<\operatorname{MSE}\left(t_{d c-1}\right)$ if

$$
\begin{aligned}
& \theta\left\{w_{6}^{2} \lambda_{004}^{*}+C_{w_{z}}^{2}\left(w_{5}^{2}-4\right)+2 w_{5} w_{6} \lambda_{003} C_{w_{z}}-\beta w_{6} \lambda_{202}^{*}-2 \alpha w_{6} \lambda_{102} C_{w_{y}}-\beta \lambda_{201} C_{w_{z}}\left(w_{5}-2\right)\right. \\
& \left.-C_{w_{y} w_{z}}\left(2 \alpha w_{5}-4 \alpha\right)\right\}-\theta^{\prime}\left\{2 \alpha C_{w_{y} w_{z}}+\beta \lambda_{201} C_{w_{z}}-3 C_{w z}^{2}\right\}+\theta^{\prime \prime}\left\{2\left(w_{2} w_{3}+w_{2} w_{5}\right) \lambda_{021} C_{w_{z}}\right. \\
& +2 \lambda_{003} C_{w_{z}}\left(w_{3} w_{4}+w_{3} w_{6}+w_{4} w_{5}\right)+\left(w_{3}^{2}+2 w_{3} w_{5}\right) C_{w_{z}}^{2}+C_{w_{x}}^{2}\left(w_{1}^{2}-1\right)+2 w_{1} w_{2} \lambda_{030} C_{w_{x}} \\
& +\left(w_{4}^{2}+2 w_{4} w_{6}\right) \lambda_{004}^{*}+2\left(w_{2} w_{4}+w_{2} w_{6}\right) \lambda_{022}^{*}+2\left(w_{1} w_{4}+w_{1} w_{6}\right) \lambda_{012} C_{w_{x}}-2 \alpha w_{4} \lambda_{102} C_{w_{y}} \\
& +w_{2}^{2} \lambda_{040}^{*}+2\left(w_{1} w_{3}+w_{1} w_{5}-4\right) C_{w_{x} w_{z}}-2 \alpha w_{3} C_{w_{y} w_{z}}-2 \alpha C_{w_{y} w_{x}}\left(w_{1}-1\right)-2 \alpha w_{2} \lambda_{120} C_{w_{y}} \\
& \left.-\beta w_{2} \lambda_{220}^{*}-\beta w_{4} \lambda_{202}^{*}-\beta w_{3} \lambda_{201} C_{w_{z}}-\beta \lambda_{210} C_{w_{x}}\left(w_{1}-1\right)\right\}<0
\end{aligned}
$$

From equations (10) and (33), $\operatorname{MSE}\left(t_{2}\right)<\operatorname{MSE}\left(t_{0}\right)$ if

$$
\begin{aligned}
& w_{4}^{2} \lambda_{004}^{*}+w_{3}^{2} C_{w_{z}}^{2}+w_{2}^{2} \lambda_{040}^{*}+w_{1}^{2} C_{w_{x}}^{2}+2 w_{1} w_{4} \lambda_{012} C_{w_{x}}+2 w_{3} w_{4} \lambda_{003} C_{w_{z}}+2 w_{2} w_{3} \lambda_{021} C_{w_{z}} \\
& +2 w_{2} w_{4} \lambda_{022}^{*}+2 w_{1} w_{3} C_{w_{x} w_{z}}-2 \alpha w_{1} C_{w_{y} w_{x}}+2 w_{1} w_{2} \lambda_{030} C_{w_{x}}-2 \alpha w_{4} \lambda_{102} C_{w_{y}}-2 \alpha w_{3} C_{w_{y} w_{z}} \\
& -2 \alpha w_{2} \lambda_{120} C_{w_{y}}-\beta w_{4} \lambda_{202}^{*}-\beta w_{3} \lambda_{201} C_{w_{z}}-\beta w_{2} \lambda_{220}^{*}-\beta w_{1} \lambda_{210} C_{w_{x}}<0
\end{aligned}
$$

From equations (19) and (33), $\operatorname{MSE}\left(t_{2}\right)<\operatorname{MSE}\left(t_{d c-2}\right)$ if

$$
\begin{aligned}
& w_{4}^{2} \lambda_{004}^{*}+C_{w_{z}}^{2}\left(w_{3}^{2}-1\right)+w_{2}^{2} \lambda_{040}^{*}+2 w_{3} w_{4} \lambda_{003} C_{w_{z}}+2 w_{2} w_{3} \lambda_{021} C_{w_{z}}+2 C_{w_{x} w_{z}}\left(w_{1} w_{3}-1\right) \\
& +2 w_{2} w_{4} \lambda_{022}^{*}+2 w_{1} w_{4} \lambda_{012} C_{w_{x}}+2 w_{1} w_{2} \lambda_{030} C_{w_{x}}-2 \alpha w_{2} \lambda_{120} C_{w_{y}}-2 \alpha C_{w_{y} w_{z}}\left(w_{3}-1\right) \\
& +C_{w_{x}}^{2}\left(w_{1}^{2}-1\right)-2 \alpha w_{4} \lambda_{102} C_{w_{y}}-2 \alpha C_{w_{y} w_{x}}\left(w_{1}-1\right)-\beta \lambda_{201} C_{w_{z}}\left(w_{3}-1\right)-\beta w_{2} \lambda_{220}^{*} \\
& -\beta w_{4} \lambda_{202}^{*}-\beta \lambda_{210} C_{w_{x}}\left(w_{1}-1\right)<0
\end{aligned}
$$

From equations (10) and (38), $M S E\left(t_{3}\right)<M S E\left(t_{0}\right)$ if equation (41) is satisfied.

From equations (22) and (38), $\operatorname{MSE}\left(t_{3}\right)<\operatorname{MSE}\left(t_{d c-3}\right)$ if equation (42) is satisfied.

\section{RESULTS AND DISCUSSION}

One artificial population and one real dataset are considered for numerical comparison of the proposed and existing estimators. The estimators are evaluated in terms of $M S E$, absolute relative bias (ARB) and percent relative efficiency $(P R E)$. Chutiman and Kumphon (2008) generated a rare and clustered population for study variable $y$ and two associated auxiliary variables $x$ and $z$. For artificial population, simulated $y, x$ and $z$ values are studied from Chutiman and Kumphon (2008). To avoid zeros, artificial population is generated using the relation,

$$
y_{i}=\left\{\begin{aligned}
4 y c_{i} & \text { if } y c_{i}>0 \\
y_{i} \sim \operatorname{Poi}(2) & \text { otherwise }
\end{aligned}\right.
$$

where $y c_{i}$ are the simulated values from Chutiman and Kumphon (2008). Auxiliary variables are generated on the same pattern. The condition of interest is $C=\{y: y>5\}$. The neighbourhood is defined as the four adjacent units. The study region is partitioned into $20 \times 20=400$ square units. The initial sample in phase one varies from 40 to 340 and in phase two, it varies from 5 to 170 . The data statistics for simulated population are: 


$$
\begin{aligned}
N & =400, \bar{Y}_{w}=8.9651, \bar{X}_{w}=5.1625, \bar{Z}_{w}=16.83, S_{w_{y}}^{2} \\
& =404.7388, S_{w_{x}}^{2}=136.0429, \\
S_{w_{z}}^{2} & =2028.643, C_{w_{y}}=2.244, C_{w_{x}}=2.2593, C_{w_{z}} \\
& =2.6762, \rho_{w_{y} w_{x}}=0.7574, \\
\rho_{w_{x} w_{z}} & =0.8806, \rho_{w_{y} w_{z}}=0.8706, C_{w_{y} w_{x}}=3.8401, C_{w_{y} w_{z}} \\
& =5.2286, C_{w_{x} w_{z}}=5.3243, \\
\lambda_{400} & =17.3587, \lambda_{040}=89.2648, \lambda_{004}=26.4474, \lambda_{300} \\
& =3.4746, \lambda_{030}=7.71, \\
\lambda_{003} & =4.2433, \lambda_{120}=3.6728, \lambda_{102}=3.4466, \lambda_{210} \\
& =2.7683, \lambda_{012}=4.3969, \\
\lambda_{201} & =3.1124, \lambda_{021}=5.4827, \lambda_{220}=17.149, \lambda_{202} \\
& =17.6509, \lambda_{022}=39.3448,
\end{aligned}
$$

For real dataset, counts of teals in $5000 \mathrm{~km}^{2}$ area, which is partitioned into $20025 \mathrm{~km}^{2}$ quadrats in central Florida are studied from Smith et al. (1995). Data on blue winged teal and green winged teal are used as auxiliary variables $x$ and $z$, respectively to generate the study variable $y$, using the model,

$$
y_{i}=4 x_{i}+4 z_{i}+e_{i}
$$

where $e_{i} \sim N\left(0, x_{i}+z_{i}\right)$. The condition of interest is $C=\{y: y>0\}$. The initial sample in phase one varies from 20 to 150 and in phase two, it varies from 2 to 75 . The data statistics for real dataset are:

$$
\begin{aligned}
N & =200, \bar{Y}_{w}=361.9401, \bar{X}_{w}=70.6048, \bar{Z}_{w}=12.01, S_{w_{y}}^{2} \\
& =3498192, S_{w_{x}}^{2}=130872.4, \\
S_{w_{z}}^{2} & =12816.53, C_{w_{y}}=5.1676, C_{w_{x}}=5.1238, C_{w_{z}} \\
& =9.4263, \rho_{w_{y} w_{x}}=0.9999, \\
\rho_{w_{x} w_{z}} & =0.5442, \rho_{w_{y} w_{z}}=0.5443, C_{w_{y} w_{x}}=26.4751, C_{w_{x} w_{z}} \\
& =26.5136,
\end{aligned}
$$

$$
\begin{aligned}
\lambda_{400} & =26.4596, \lambda_{040}=26.4355, \lambda_{004}=96.9604, \lambda_{300} \\
& =5.0454, \lambda_{030}=5.0418, \\
\lambda_{003} & =9.7831, \lambda_{120}=5.0430, \lambda_{102}=5.1769, \lambda_{210} \\
& =5.0442, \lambda_{012}=5.1757, \\
\lambda_{201} & =2.7462, \lambda_{021}=2.7450, \lambda_{220}=26.4475, \lambda_{202} \\
& =27.1226, \lambda_{022}=27.1103,
\end{aligned}
$$

The results for MSE, ARB and PRE of different estimators for estimation of population mean, variance and coefficient of variation are presented in Tables 1,2 and 3, respectively. The estimators are proposed under three different situations, i.e. partial, no and full information about parameters of auxiliary variable. All the results reveal the same pattern for both artificial population and real dataset. The proposed estimators for population mean in three different situations are $t_{1 m}, t_{2 m}, t_{3 m}$. Similarly estimators proposed for population variance and coefficient of variation are $t_{1 v}$, $t_{2 v}, t_{3 v}$ and $t_{1 c}, t_{2 c}, t_{3 c}$, respectively. For small initial sample size, the proposed estimators are highly efficient with some amount of $A R B$ present but as initial sample size increases, the proposed estimators are efficient with vanishing $A R B$. The proposed estimators for population mean, variance and coefficient of variation have high PRE (less MSE) than the existing estimators in all three situations. As initial sample size increases, PRE of proposed estimators for population mean, variance and coefficient of variation increases for situations 1 and 2 and remains constant for situation 3 .

\section{CONCLUSION}

Exponential ratio type estimators are proposed for the general parameter $\left(\tau_{(\alpha, \beta)}=\bar{Y}_{w}^{\alpha} S_{w_{v}}^{\beta}\right)$ using two auxiliary variables in adaptive cluster sampling under two phase sampling schemes. The results from both populations depict that the proposed estimators for general parameter are more efficient than the usual estimator proposed by Thompson (1990) and the ratio type estimator proposed by Dryver and Chao (2007) in all three situations. This shows that use of both population mean and variance of auxiliary variables is beneficial. 


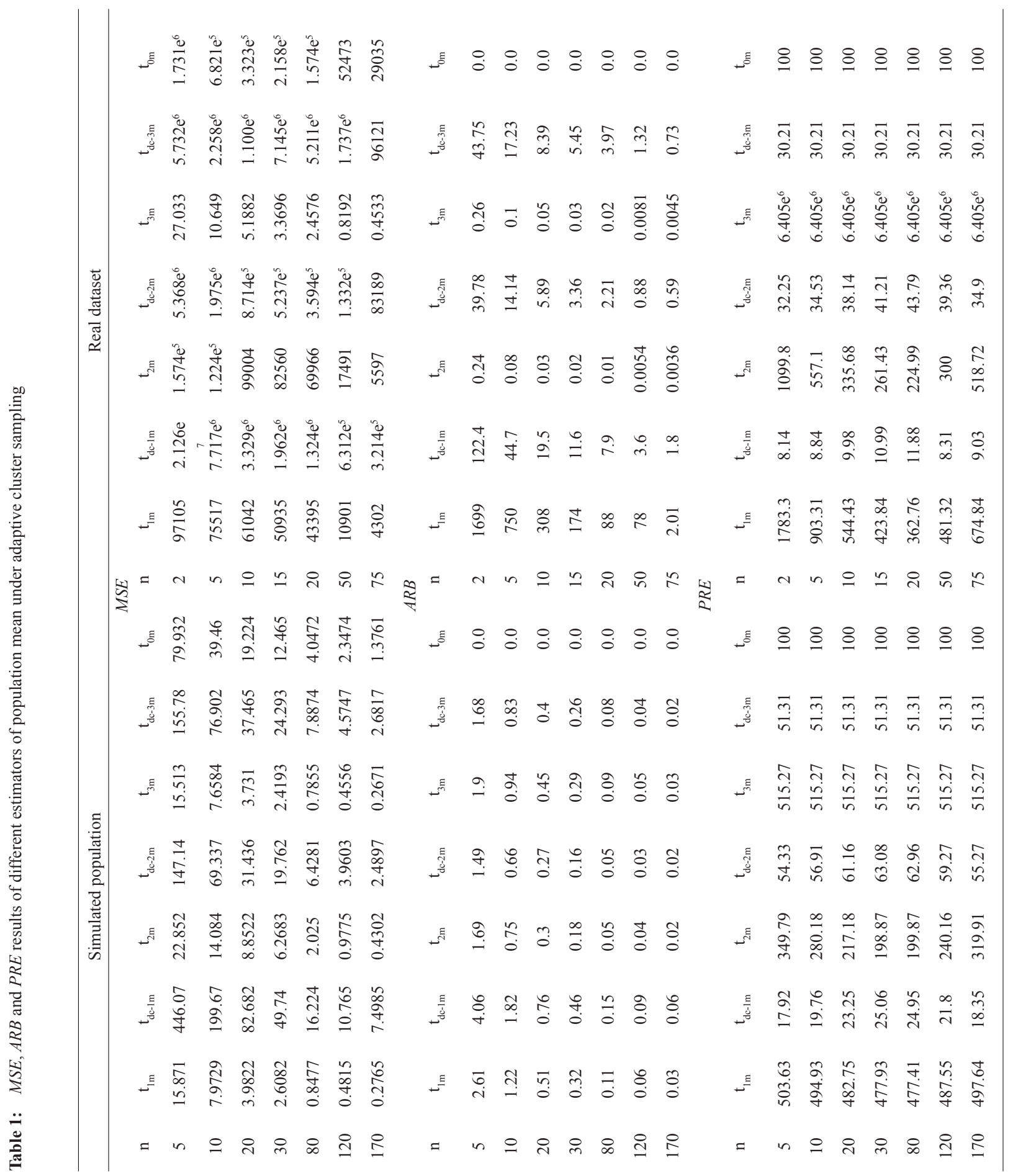




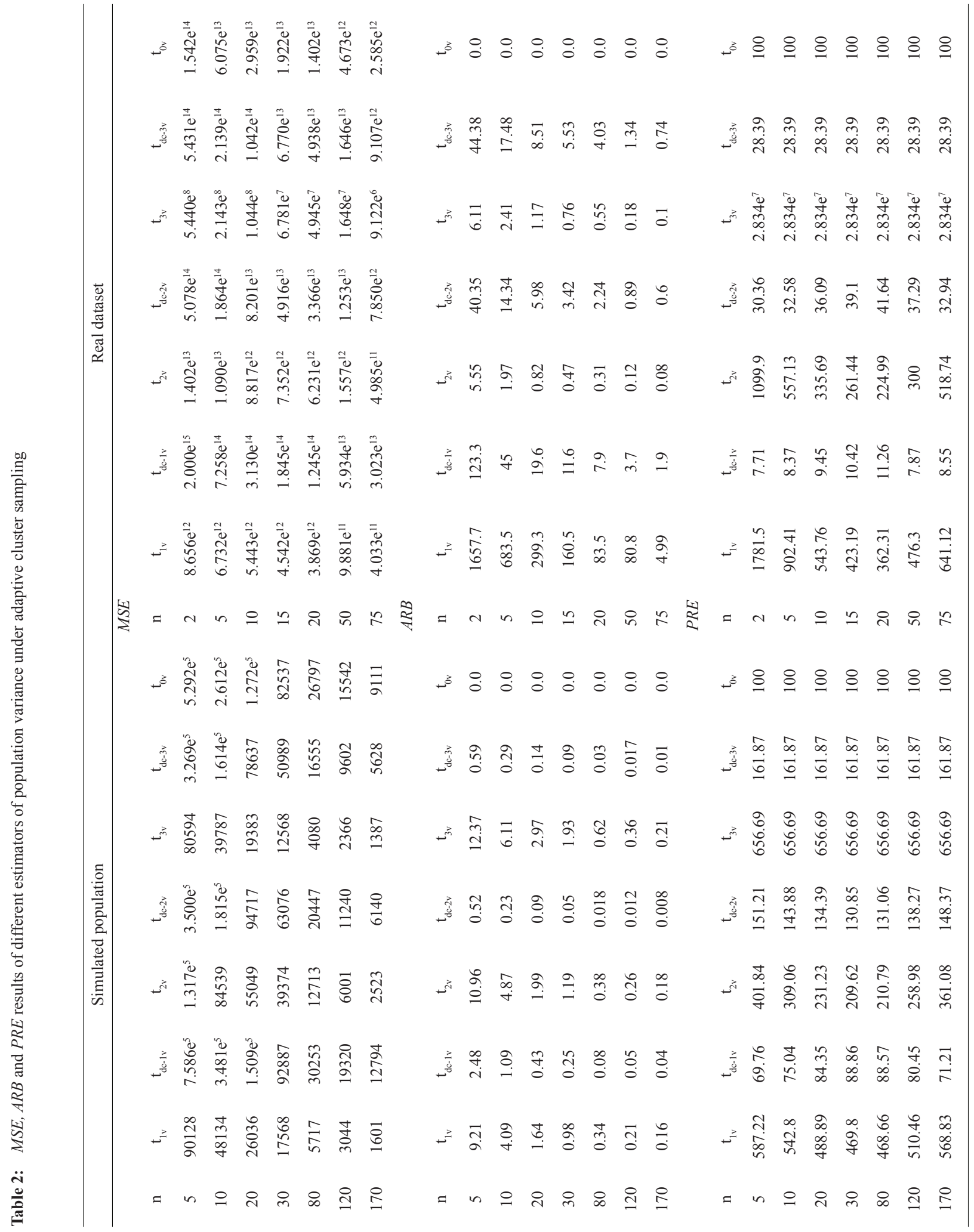




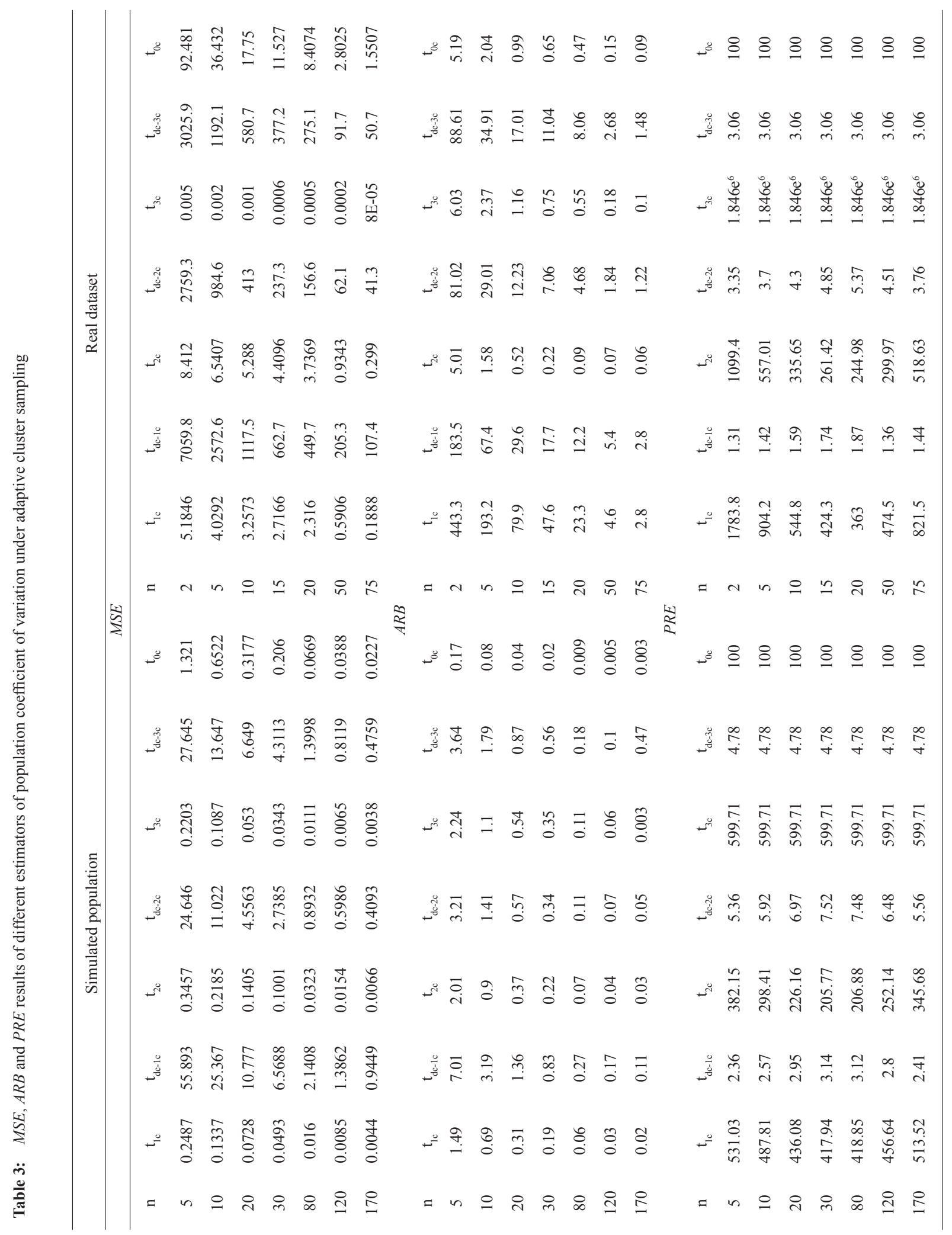




\section{REFERENCES}

Chutiman N. \& Kumphon B. (2008). Ratio estimators using two auxiliary variables for adaptive cluster sampling. Thailand Statistician 6: $241-256$.

Dryver A.L. \& Chao C.T. (2007). Ratio estimators in adaptive cluster sampling. Environmetrics 18: 607 - 620 .

DOI: https://doi.org/10.1002/env.838

Shabbir J. \& Gupta S. (2017). Estimation of population coefficient of variation in simple and stratified random sampling under two-phase sampling scheme when using two auxiliary variables. Communications in Statistics -
Theory and Methods 46: 8113 - 8133 .

DOI: https://doi.org/10.1080/03610926.2016.1175627

Smith D.R., Conroy M.J. \& Brakhage D.H. (1995). Efficiency of adaptive cluster sampling for estimating density of wintering waterfowl. Biometrics 51(2): 777 - 788.

DOI: https://doi.org/10.2307/2532964

Thompson S.K. (1990). Adaptive cluster sampling. Journal of the American Statistical Association 85: $1050-1059$.

DOI: https://doi.org/10.1080/01621459.1990.10474975

Thompson S.K. (1992). Sampling. John Wiley \& Sons Inc., New York, USA. 